\title{
Comunicação
}

[Comunication]

\section{Ocorrência de não conformidades físico-químicas e microbiológicas em leite e derivados no estado de Minas Gerais, no período de 2011 a 2015}

\author{
[Occurrence of physico-chemical and microbiological nonconformities in milk \\ and dairy products in the State of Minas Gerais, from 2011 to 2015] \\ V.C. Santos ${ }^{1}$, D.C.S.Z. Ribeiro ${ }^{2}$, L.M. Fonseca ${ }^{3} *$ \\ ${ }^{1}$ Instituto Mineiro de Agropecuária - Belo Horizonte, MG \\ ${ }^{2}$ Aluna de pós-graduação - Escola de Veterinária - Universidade Federal de Minas \\ Gerais - Belo Horizonte, MG \\ ${ }^{3}$ Escola de Veterinária - Universidade Federal de Minas Gerais - Belo Horizonte, MG
}

O Brasil é o quinto maior produtor de leite do mundo, com aproximadamente 33,5 bilhões de litros em 2017. O estado de Minas Gerais é o maior produtor nacional e contribui com cerca de 9 bilhões de litros de leite por ano, com o segundo maior rebanho bovino do Brasil (Pesquisa..., 2018).

Uma crescente demanda do mercado consumidor por produtos de alta qualidade, que mantenham suas características, revela a necessidade de análises que garantam a segurança microbiológica na produção e a vida de prateleira do produto. O setor laticinista também demanda produtos lácteos de alta qualidade, levando à progressiva adaptação desse importante segmento às exigências do mercado consumidor (Oliveira et al., 2012). A qualidade e o maior rendimento de produtos lácteos estão intimamente relacionados à qualidade da matéria-prima, desde a sua obtenção até a sua entrada na linha de processamento (Teodoro e Oliveira, 2015). E, para monitoramento dessa qualidade, é necessário que o serviço de inspeção realize análises que comprovem a composição e a segurança do produto final.

Vários surtos e casos de intoxicação e/ou infecção têm sido reportados, muitos associados ao leite e aos seus derivados. O sistema de notificação no Brasil ainda é bastante incipiente e os registros não representam a realidade nacional. Isso ocorre porque a probabilidade de um surto ser reconhecido como tal e notificado

Recebido em 1 de outubro de 2018

Aceito em 9 de abril de 2019

* Autor para correspondência (corresponding author)

E-mail: leorges@gmail.com pelas autoridades competentes depende, dentre outros fatores, da notificação pelos consumidores, do relato dos médicos, das atividades de vigilâncias sanitária e epidemiológica das Secretarias Municipais e Estaduais de Saúde. Os dados epidemiológicos de surtos de origem alimentar ocorridos no país são escassos, desatualizados e de difícil acesso. Somente cerca de $10 \%$ do total de surtos é notificado às autoridades competentes, devido à falha nos sistemas de notificação e de fiscalização (Forsythe, 2002; Rahimi et al., 2010).

$\mathrm{O}$ poder público responde à crescente demanda dos consumidores pela segurança alimentar e melhoria da qualidade por meio da regulamentação de regras que visam ao controle das etapas do processamento dos alimentos, com o objetivo de garantir inocuidade, integridade e aperfeiçoamento dos processos de produção. Um dos principais parâmetros utilizados para se verificar a qualidade dos produtos de origem animal é o perfil microbiológico, visto que a presença de contaminação microbiana em leite e em seus derivados pode indicar risco higiênicosanitário e comprometimento da vida de prateleira, já que promove a deterioração de seus componentes (Chambers, 2002).

A utilização da contagem total de aeróbios mesófilos como indicador de qualidade pode indicar deficiências na sanitização ou falha no controle do processo ou dos ingredientes. Por exemplo, aplicada a ingredientes, esta avaliação deve levar em conta a diluição e o efeito no 
produto final. Aplicada a alimentos desidratados, pode indicar se o controle da umidade está sendo corretamente aplicado ao processo de secagem (Silva et al., 2001). Altas contagens de coliformes em lácteos também está associada às condições inadequadas de higiene durante a ordenha de leite, o processamento e a estocagem dos lácteos, ou, ainda, à recontaminação após tratamento térmico (Felício et al., 2013). Portanto, a presença de coliformes em alimentos processados é considerada uma indicação útil de contaminação pós-sanitização ou pós-processo, evidenciando práticas de higiene e sanitização aquém dos padrões requeridos para o processamento de alimentos (Silva et al., 2001).

Bolores e leveduras são muito disseminados na natureza e encontram no ambiente das indústrias lácteas condições favoráveis para o seu desenvolvimento, mesmo em produtos com média ou baixa umidade. O queijo é um ótimo substrato para o desenvolvimento de bolores. Portanto, os bolores são importantes agentes de deterioração desses alimentos (Nicolau, 2000). O tratamento térmico do leite é de fundamental importância para a obtenção de um produto com qualidade. A eficiência do processo de pasteurização pode ser avaliada por meio do binômio tempo e temperatura, resultando na inativação da atividade enzimática da fosfatase alcalina e na manutenção da atividade da peroxidase do leite (Brasil, 2017). O controle rigoroso do tratamento térmico integra a aplicação das boas práticas de fabricação no âmbito da indústria.

O objetivo do presente estudo foi diagnosticar, por meio da avaliação de dados oficiais, a ocorrência de não conformidades microbiológicas e físico-químicas de leite e derivados lácteos produzidos em indústrias laticinistas registradas no Instituto Mineiro de Agropecuária, no período de 2011 a 2015. Os produtos analisados foram coletados em indústrias registradas no Instituto Mineiro de Agropecuária (IMA). As análises foram realizadas no Laboratório de Química Agropecuária do IMA, localizado na Ceasa MG, no município de Contagem. Fiscais Agropecuários (médico veterinário) e/ou fiscais assistentes (técnico agropecuário) coletaram duas amostras distintas de cada produto, que foram enviadas ao laboratório, segundo o Manual de Procedimentos de Fiscalização (Minas Gerais,
2007). Os resultados analíticos foram compilados em um banco de dados e cedidos pela Gerência de Inspeção de Produtos (GIP) do IMA, totalizando 5421 amostras analisadas por meio de técnicas analíticas padronizadas (192 em 2011, 1008 em 2012, 1368 em 2013, 1271 em 2014, 1582 em 2015).

Os produtos analisados incluíram 863 amostras de leite pasteurizado, 21 de creme de leite, 318 de doce de leite (pastoso e em barra), 278 de manteiga (comum e de primeira qualidade), 1816 de muçarela, 423 de queijo minas frescal, 224 de queijo minas padrão, 420 de ricota fresca, 18 de coalhada e 552 de iogurte. Uma alíquota de cada produto amostrado foi enviada para o Laboratório de Segurança Microbiológica em Alimentos, para a realização das análises microbiológicas de Listeria monocytogenes, Salmonella spp., Staphylococcus coagulasepositiva, bolores e leveduras, coliformes a $35^{\circ} \mathrm{C}$ e coliformes a $45^{\circ} \mathrm{C}$ e contagem de células viáveis. A outra alíquota foi enviada para realização das análises físico-químicas no Laboratório de Análises Físico-Químicas em Alimentos (LFQA), onde foram determinados a fosfatase residual, a peroxidase, a acidez, a acidez na gordura, o extrato seco desengordurado, a matéria gorda no extrato seco, a matéria gorda láctea, a proteína, a umidade, o índice crioscópico, o nitrato, o amido, os nutralizantes da acidez e o peróxido. Os padrões físico-químicos e microbiológicos dos produtos analisados e os procedimentos referentes às exigências de análises laboratoriais observaram os parâmetros especificados na Portaria 1309, de 14 de maio de 2013 (Minas Gerais, 2013). A análise estatística foi descritiva e a apresentação dos resultados foi pela frequência das não conformidades encontradas.

Os resultados totais indicaram que $2010(37,1 \%)$ amostras de leite pasteurizado e de derivados lácteos apresentaram não conformidade à legislação em vigor, sendo 1149 relacionadas às análises físico-químicas e 861 às análises microbiológicas. Os resultados das análises microbiológicas estão apresentados na Tab. 1. Os coliformes a $35^{\circ} \mathrm{C}$ e os termotolerantes $\left(45^{\circ} \mathrm{C}\right)$ apresentaram valores elevados. De acordo com os padrões legais, 65 amostras de leite pasteurizado não estariam em conformidade com a IN 62 (Brasil, 2011), legislação vigente no período das análises. Esse achado reforça a 
hipótese de falha na monitorização da pasteurização quando associada ao resultado positivo para a pesquisa de fosfatase alcalina.

A mensuração do número de microrganismos aeróbios mesófilos ou contagem padrão em placas do leite pasteurizado também auxilia na avaliação da qualidade e de sua conservação, bem como na eficiência da pasteurização. Em 32 amostras de leite pasteurizado, o resultado foi maior do que $8,0 \times 10^{4} \mathrm{UFC} / \mathrm{mL}$. Correlacionando o resultado com a pesquisa de fosfatase alcalina positiva, pôde-se indicar, novamente, falhas de tratamento térmico ou recontaminação (Jay et al., 2005).

O número de amostras contaminadas por bolores e leveduras totalizou 187, abrangendo sete diferentes produtos. Para Staphylococcus coagulase-positiva, houve 118 amostras não conformes aos padrões legais, sendo 80 amostras de muçarela. Tais valores são considerados extremamente elevados, sobretudo por se tratar de produtos que, teoricamente, foram submetidos ao tratamento térmico. Especificamente, no produto muçarela, essas contagens são preocupantes sob o ponto de vista da saúde pública, por estarem correlacionadas à detecção da atividade da fosfatase alcalina, o que corrobora $o$ fato de que nem sempre o aquecimento durante a filagem é suficiente para eliminação de microrganismos patogênicos. Outra potencial fonte de contaminação por $S$. aureus é a manipulação do queijo após a filagem para moldagem (Dias et al., 2012).

Salmonella spp. foram identificadas em uma amostra de leite pasteurizado, uma de queijo minas frescal e duas de manteiga. Dentre as possíveis causas, destacam-se a contaminação pela água, por recipientes contaminados e pela manipulação humana durante $\mathrm{o}$ envase. $\mathrm{O}$ resultado positivo para a fosfatase sugere negligência no controle do tratamento térmico do leite, pois o leite cru está frequentemente associado aos principais surtos causados por esta bactéria.

Tabela 1. Resultados das análises microbiológicas de leite e de derivados lácteos em análise fiscal pelo IMA, de 2011 a 2015

\begin{tabular}{|c|c|c|c|c|c|c|c|c|c|}
\hline Produto & Situação & $\mathrm{n}$ & $\begin{array}{c}\text { Coliformes } \\
\text { a } 35^{\circ} \mathrm{C}\end{array}$ & $\begin{array}{c}\text { Coliformes } \\
\text { a } 45^{\circ} \mathrm{C}\end{array}$ & Mesófilos & $\begin{array}{c}\text { Bolores e } \\
\text { Leveduras }\end{array}$ & $\begin{array}{c}\text { Staphylococcus } \\
\text { coagulase } \\
\text { positiva }\end{array}$ & $\begin{array}{l}\text { Salmonella } \\
\text { spp. }\end{array}$ & $\begin{array}{c}\text { Total } \\
\text { de } \\
\text { NC } \\
\end{array}$ \\
\hline \multirow[t]{2}{*}{ Leite pasteurizado } & $\mathrm{C}$ & 402 & 0 & 0 & 0 & $*$ & $*$ & 0 & \\
\hline & Total & 863 & 55 & 65 & 32 & $*$ & $*$ & 1 & \\
\hline \multirow[t]{2}{*}{ Iogurte } & $\mathrm{C}$ & 262 & 0 & 0 & $*$ & 0 & $*$ & $*$ & \\
\hline & $\mathrm{NC}$ & 290 & 6 & 6 & $*$ & 33 & $*$ & $*$ & 45 \\
\hline \multirow{2}{*}{ Creme de leite } & $\mathrm{NC}$ & 2 & $*$ & $*$ & 1 & $*$ & $*$ & $*$ & 1 \\
\hline & Total & 21 & $*$ & $*$ & 1 & $*$ & $*$ & $*$ & \\
\hline \multirow{3}{*}{ Doce de leite } & $\mathrm{C}$ & 200 & $*$ & $*$ & $*$ & 0 & $*$ & $*$ & \\
\hline & $\mathrm{NC}$ & 118 & $*$ & $*$ & $*$ & 6 & $*$ & $*$ & 6 \\
\hline & Total & 318 & $*$ & $*$ & $*$ & 6 & $*$ & $*$ & \\
\hline Manteiga & $\mathrm{C}$ & 135 & 0 & 0 & $*$ & 0 & 0 & 0 & \\
\hline \multirow{2}{*}{ Queijo minas frescal } & $\mathrm{NC}$ & 325 & 66 & 30 & $*$ & 55 & 12 & 1 & 164 \\
\hline & Total & 423 & 66 & 30 & $*$ & 55 & 12 & 1 & \\
\hline \multirow[t]{3}{*}{ Queijo minas padrão } & $\mathrm{C}$ & 136 & $*$ & $*$ & $*$ & $*$ & 0 & $*$ & \\
\hline & $\mathrm{NC}$ & 88 & $*$ & $*$ & $*$ & $*$ & 14 & $*$ & 14 \\
\hline & Total & 224 & $*$ & $*$ & $*$ & $*$ & 14 & $*$ & \\
\hline \multirow[t]{3}{*}{ Muçarela } & $\mathrm{C}$ & 1587 & 0 & 0 & $*$ & 0 & 0 & $*$ & \\
\hline & $\mathrm{NC}$ & 229 & 9 & 33 & $*$ & 10 & 80 & $*$ & 132 \\
\hline & Total & 1816 & 9 & 33 & $*$ & 10 & 80 & $*$ & \\
\hline \multirow[t]{3}{*}{ Ricota } & $\mathrm{C}$ & 264 & $*$ & $*$ & $*$ & 0 & $*$ & $*$ & \\
\hline & $\mathrm{NC}$ & 156 & $*$ & $*$ & $*$ & 68 & $*$ & $*$ & 68 \\
\hline & Total & 420 & $*$ & $*$ & $*$ & 68 & $*$ & $*$ & \\
\hline Coalhada & $\mathrm{C}$ & 8 & $*$ & $*$ & $*$ & 0 & $*$ & $*$ & \\
\hline
\end{tabular}

Análises realizadas segundo Portaria 1309, de 14/05/2013 (Minas Gerais, 2013). C= conforme; NC= não conforme. 
Os resultados das análises físico-químicas estão apresentados na Tab. 2. Os teores de sólidos não gordurosos (SNG) de 49 amostras de leite pasteurizado foram inferiores a $8,4 \mathrm{~g} / 100 \mathrm{~g}$. A gordura foi o componente sólido que apresentou maior número de não conformidades no leite pasteurizado, com 37 amostras abaixo do valor de 3\% estabelecido pela legislação (Brasil, 2018). Iogurte, doce de leite, manteiga e queijo minas frescal também apresentaram amostras não conformes para teor de gordura. Um total de sete amostras de leite pasteurizado não atendeu ao padrão de acidez situado entre 0,14 e 0,18 gramas de ácido láctico/100mL (Brasil, 2018). Problemas de nutrição e manejo do rebanho, contaminação do leite ou a ocorrência de fraudes (adição de água e adição de substâncias neutralizantes, por exemplo) podem resultar na alteração da acidez do leite. Quanto ao iogurte, 14 amostras não atenderam ao padrão de acidez titulável de 0,6 a 1,5g de ácido láctico/100g, valor estabelecido pela legislação (Brasil, 2007). Para o teor de proteína, 164 amostras analisadas estavam não conformes. Das amostras analisadas de leite pasteurizado, 86 não atenderam ao padrão para o índice crioscópico, o que indica fraude do leite com adição de água. A umidade também foi não conforme em outros nove produtos.

Do total de amostras analisadas de leite pasteurizado, 16 apresentaram resultado positivo na pesquisa de fosfatase alcalina. Ainda apresentaram fosfatase positiva 78 amostras de muçarela e amostras de outros 72 produtos. A pesquisa da enzima fosfatase alcalina é prova de controle da pasteurização do leite, realizada após o processamento que inativa sua atividade enzimática. Uma prova de fosfatase positiva é indicativo de subaquecimento durante $o$ processamento térmico ou mistura de leite cru no leite pasteurizado. Para a pesquisa de peroxidase, 33 amostras de leite pasteurizado apresentaram resultado negativo de atividade enzimática. A pesquisa da peroxidase é utilizada para verificar o superaquecimento do leite, uma vez que a enzima é inativada em condições de temperatura superiores aos da pasteurização.

Tabela 2. Amostras não conformes das análises físico-químicas de leite e de derivados lácteos em análise fiscal pelo IMA, de 2011 a 2015

\begin{tabular}{|c|c|c|c|c|c|c|c|c|c|c|c|c|c|}
\hline $\begin{array}{l}\text { Análise } \\
\text { produto }\end{array}$ & $\mathrm{n}$ & SNG & G & A & $\mathrm{P}$ & IC & $\mathrm{U}$ & FA & $\mathrm{PE}$ & $\mathrm{AM}$ & $\mathrm{Cl}$ & NA & $\mathrm{P}$ \\
\hline $\begin{array}{l}\text { Leite } \\
\text { pasteurizado }\end{array}$ & 863 & 49 & 37 & 7 & 2 & 86 & & 16 & 33 & & 2 & 4 & 1 \\
\hline Manteiga & 278 & 1 & 24 & & & & 36 & & & & & & \\
\hline Doce de leite & 318 & & 31 & & 26 & & 48 & & & 1 & & & \\
\hline Iogurte & 552 & & 66 & 14 & 127 & & & & & 29 & & & \\
\hline $\begin{array}{l}\text { Queijo minas } \\
\text { frescal }\end{array}$ & 423 & & 29 & & & & 8 & 32 & & & & & \\
\hline Queijo prato & 46 & & & & & & 4 & 3 & & & & & \\
\hline $\begin{array}{l}\text { Queijo minas } \\
\text { meia-cura }\end{array}$ & 10 & & & & & & 1 & 2 & & & & & \\
\hline $\begin{array}{l}\text { Queijo minas } \\
\text { padrão }\end{array}$ & 224 & & & & & & & 26 & & & & & \\
\hline Coalhada & 18 & & & & 2 & & & & & & & & \\
\hline $\begin{array}{l}\text { Bebida láctea } \\
\text { fermentada }\end{array}$ & 227 & & & & 6 & & & & & & & & \\
\hline Bebida láctea & 52 & & & & 1 & & & & & & & & \\
\hline $\begin{array}{l}\text { Requeijão } \\
\text { cremoso }\end{array}$ & 187 & & & & & & 23 & & & & & & \\
\hline Parmesão & 77 & & & & & & 17 & & & & & & \\
\hline Muçarela & 1816 & & & & & & 3 & 78 & & & & & \\
\hline Ricota & 420 & & & & & & 1 & 3 & & & & & \\
\hline Provolone & 170 & & & & & & & 6 & & & & & \\
\hline
\end{tabular}

SNG=sólidos não gordurosos; G=gordura; A=acidez; P=proteína; IC=índice crioscópico; U=umidade; FA=fosfatase alcalina; $\mathrm{PE}=$ peroxidase enzima; $\mathrm{AM}=$ amido; $\mathrm{Cl}=$ cloreto; $\mathrm{NA}=$ neutralizantes da acidez; $\mathrm{P}=$ peróxido. $\mathrm{n}$ (número de amostras analisadas) $=5421$. 
As pesquisas de amido apresentaram resultado positivo em 31 amostras de três produtos (doce de leite, iogurte e bebida láctea fermentada). Porém, como o uso desse ingrediente em certos produtos é permitido dentro dos limites legais, provas qualitativas não são conclusivas, exceto se o ingrediente não estiver previsto no rótulo. Entre as amostras de leite pasteurizado, quatro apresentaram neutralizantes da acidez, duas com amido e uma com peróxido de hidrogênio. Qualquer substância adicionada ao leite caracteriza-se fraude/adulteração e está em desacordo com a legislação (Brasil, 2017).

Para todos os parâmetros microbiológicos avaliados, ocorreram não conformidades, revelando possivelmente falhas de higienização do ambiente e de equipamentos. Além disso, a contaminação do leite pós-pasteurização, a utilização de fermentos inativos, de temperaturas inadequadas e incorretas condições de manufatura e armazenagem contribuem também, de forma efetiva, para o comprometimento da qualidade do produto final, conforme descrito por Jay et al. (2005).
Os resultados revelaram deficiência na qualidade higiênico-sanitária e irregularidades na composição dos produtos, o que provavelmente está relacionado à qualidade da matéria-prima empregada na fabricação e no processamento. Destaca-se a ocorrência de não conformidades relacionadas à presença da atividade da fosfatase alcalina, o que indica a utilização de leite cru e o consequente risco à saúde pública. Tal ocorrência no queijo muçarela é indicativo da insuficiência do aquecimento durante a filagem, quando da utilização do leite cru para a fabricação desse queijo. É importante salientar que esses resultados indicam risco potencial à saúde da população e uma necessidade da aplicação efetiva das boas práticas de fabricação, além da permanente ação fiscalizadora.

Palavras-chave: leite pasteurizado, qualidade de produtos lácteos, análise físico-química e microbiológica, não conformidades, inspeção de alimentos

\begin{abstract}
Milk and dairy products are potential sources for spoilage and pathogenic microorganisms, and although a huge amount of data is collected by the official inspection services, only a few reports are available to the public. This study aimed to evaluate the data for physicochemical and microbiological quality of pasteurized milk and dairy products, collected for inspection at industrial establishments registered at the Instituto Mineiro de Agropecuária (IMA), which is an official inspection service in Minas Gerasi State, Brazil. A total of 192 analyzes were done in 2011, 1008 in 2012, 1368 in 2013, 1271 in 2014, 1582 in 2015, adding up to 5421 samples analyzed by standard analytical techniques in official government laboratories. The statistical analysis was descriptive. A total of 2010 analytical results were nonconform to the legal requirements. Among the results, 78 (4.3\%) samples of mozzarella cheese were positive for alkaline phosphatase, and freezing point results for pasteurized milk were outside the legal requirements in $86(10 \%)$ samples. Staphylococcus coagulase positive was above limits in 80 (4.4\%) samples of mozzarella cheese. These results indicate a risk to the consumer's health even in pasteurized products, and the need for effective enforcement of good manufacturing practices in the food industries.
\end{abstract}

Keywords: pasteurized milk, dairy products quality, physicochemical and microbiological analysis, nonconformities, food inspection

\section{AGRADECIMENTOS}

Coordenação de Aperfeiçoamento de Pessoal de Nível Superior - Capes, pelo suporte financeiro; Fapemig e Instituto Mineiro de Agropecuária.

\section{REFERENCIAS}

BRASIL. Ministério da Agricultura e do Abastecimento. Instrução Normativa n.46, de 23/10/2007. Regulamento técnico de identidade e qualidade de leites fermentados. Diário Oficial da União, Brasília, 24 out. 2007. 
BRASIL. Ministério da Agricultura, Pecuária e Abastecimento. Decreto n. 9013, de 29/03/2017. Regulamenta a Lei n.1283, de 18 de dez. de 1950, e a Lei n.7889, de 23 de nov. de 1989, que dispõem sobre a inspeção industrial e sanitária de produtos de origem animal. Diário Oficial da União, Brasília, 30 mar. 2017.

BRASIL. Ministério da Agricultura, Pecuária e Abastecimento. Instrução Normativa n.62, de 29/12/2011. Aprova o regulamento técnico de identidade e qualidade do leite tipo $\mathrm{A}$, o regulamento técnico de identidade e qualidade do leite cru refrigerado, o regulamento técnico de identidade e qualidade do leite pasteurizado e o regulamento técnico da coleta de leite cru refrigerado e seu transporte a granel. Diário Oficial da União, Brasília, 30 dez. 2011.

BRASIL. Ministério da Agricultura, Pecuária e Abastecimento. Instrução Normativa n.76, de $26 / 11 / 2018$. Aprova os regulamentos técnicos que fixam a identidade e as características de qualidade que devem apresentar o leite cru refrigerado, o leite pasteurizado e o leite pasteurizado tipo A, na forma desta Instrução Normativa e do Anexo Único. Diário Oficial da União, Brasília, 30 nov. 2018.

CHAMBERS, J.V. The microbiology of raw milk. In: ROBINSON, R.K. (Ed.). Dairy Microbiology Handbook. New York: WileyInterscience, 2002. p.39-90.

DIAS, M.A.C.; SANT'ANA, A.S.; CRUZ, A.G. et al. On the implementation of good manufacturing practices in a small processing unity of mozzarella cheese in Brazil. Food Control, v.24, n.1/2, p.199-205, 2012.

FELÍCIO, B.A.; LEMPK, M.W.; PINTO, M.S. et al. Efeito in vitro de nisina sobre pool de Staphylococcus aureus isolados de Queijo Minas artesanal da região do Campo das Vertentes MG. Rev. Instit. Latic. Cândido Tostes, v.68, p.19-23, 2013.

FORSYTHE, S.J. Microbiologia da segurança alimentar. Porto Alegre: Artmed, 2002. 424p.
JAY, J.M.; LOESSNER, M.J.; GOLDEN, D.A Modern food microbiology. 7.ed. New York: Springer, 2005. 790p.

MINAS GERAIS. Portaria 1309 de 14 maio de 2013. Dispõe sobre os parâmetros e padrões físico-químicos e microbiológicos de produtos de origem animal e água de abastecimento. Belo Horizonte, Instituto Mineiro de Agropecuária IMA, 14 de mai. 2013.

MINAS GERAIS. Portaria 832 de 12 de março de 2007. Manual de procedimentos de fiscalização de produtos de origem animal. Belo Horizonte, Instituto Mineiro de Agropecuária IMA, 12 de mar. 2007.

NICOLAU, E.S. Avaliação das condições higiênico-sanitárias de indústria de laticínios produtoras de queijo tipo mussarela na região de Goiania-GO, com ênfase para o Staphylococcus aureus. 2000. 109f. Tese (Doutorado em Tecnologia de Alimentos) Universidade Estadual de Campinas, Campinas, SP.

OLIVEIRA, M.C.P.P.; SILVA, N.M.A.; BASTOS, L.P.F. et al. Fourier Transform Infrared Spectroscopy (FTIR) for MUN analysis in normal and adulterated milk. Arq. Bras. Med. Vet. Zootec., v.64, p.1360-1366, 2012.

PESQUISA trimestral do leite. Rio de Janeiro: IBGE, 2018. Disponível em: <https://www.ibge.gov.br/estatisticasnovoportal/economicas/agricultura-epecuaria/9209-pesquisa-trimestral-do-leite.html> Acessado em 28 set 2018

RAHIMI, E.; AMERI, M.; MONTAZ, H. Prevalence and antimicrobial resistence of Listeria species isolated from Milk and dairy products in Iran. Food Control, v.21, p.14481442, 2010.

SILVA, N.; JUNQUEIRA, V.C.A.; SILVEIRA, N.F. Manual de métodos de análises microbiológicas de alimentos. São Paulo: Varela, 2001. 317p.

TEODORO, V.A.M.; OLIVEIRA, P.H.B. Exigências legais para registro de indústrias de laticínios. Inf. Agropecu., v.36, p.20-35, 2015. 